\title{
Détermination de l'âge osseux chez les migrants est inconvenable
}

\author{
Georg Friedrich Eicha, Valérie Schwitzgebel ${ }^{b}$ \\ a PD Dr med., Président de la Société Suisse de Radiologie Pédiatrique (SSRP) \\ b Prof. Dr med., Présidente de la Société Suisse d'Endocrinologie et Diabétologie Pédiatriques (SSEDP)
}

Les bouleversements politiques actuels et les guerres dans le monde, en particulier au Proche Orient et en Afrique du Nord, ont pour conséquence l'arrivée en Europe de nombreux réfugiés, ce qui concerne aussi la Suisse. Parmi les réfugiés se trouvent beaucoup d'enfants et d'adolescents dont certains ne sont pas accompagnés, et de jeunes adultes qui ne possèdent souvent pas de documents d'identité ou des documents d'identification incomplets. La date et le lieu de naissance sont donc souvent des données manquantes. Il faut savoir que les requérants d'asile mineurs bénéficient d'une protection et d'une assistance particulière. L'âge exact (l'âge chronologique) d'un réfugié a une importance juridique considérable. La détermination radiologique de l'âge osseux pour différencier les mineurs des majeurs est de plus en plus sollicitée par les autorités impliquées dans le processus d'asile. Un examen correspondant, c'est-à-dire une radiographie de la main est effectuée par les médecins et évaluée selon l'Atlas de Greulich et Pyle [1]. L'utilisation de ce processus pour déterminer l'âge chronologique des requérants d'asile est, à notre avis, problématique [2-4]. Nous pensons qu'il serait préférable de ne plus pratiquer cet examen radiologique chez les réfugiés pour les raisons suivantes.

1. La détermination de l'âge osseux selon Greulich et Pyle ou encore Tanner \& Whitehouse sont des méthodes standard reconnues afin de déterminer l'âge biologique. Elles ne sont pas conçues pour déterminer l'âge chronologique. Elles n'ont pas été testées sur ce point.

2. La variabilité individuelle (l'écart-type) de l'âge osseux, selon Greulich et Pyle, par exemple pour un jeune homme de 17 ans est de 15,4 mois. En tenant compte d'un double écart-type qui est couramment utilisé pour mesurer la norme en médecine, il en résulte une différence de plus de 2 ans. De ce fait, un garçon de 17 ans en bonne santé peut avoir un âge osseux de 19 ans et donc une maturation osseuse terminée. En d'autres termes: même avec la fusion complète du cartilage de croissance il est possible que l'âge chronologique soit de moins de 18 ans.
3. Les filles atteignent la fin de leur croissance plus tôt que les garçons ce qui augmente la possibilité que l'examen radiologique de la main d'une fille mineure révèle un squelette mature suggérant un âge plus avancé.

4. La méthode de Greulich et Pyle est basée sur un collectif normal d'enfants d'origine européenne des Etats-Unis. Cette étude a été réalisée au début du $20^{\mathrm{e}}$ siècle. Les données actuelles concernant l'âge osseux d'ethnies différentes ne sont disponibles que ponctuellement et ne peuvent d'autant moins être appliquées à la population actuelle de migrants [5].

5. Certaines maladies comme les troubles du système endocrinien peuvent interférer avec le processus de maturation osseuse. Ainsi, une évaluation de la maturation osseuse sans examen médical supplémentaire et/ou sans évaluer l'état nutritionnel doit être rejetée.

6. La radiographie est réalisée avec des rayons ionisants, potentiellement cancérigènes. Bien que la dose de radiation pour la radiographie de la main soit relativement faible et ne correspond qu'à une fraction de la dose annuelle naturelle, cet examen n'est effectué que dans le cadre clinique pour des indications médicales. Ceci ne s'applique pas aux réfugiés.

Nous concluons donc que l'évaluation de l'âge osseux des requérants d'asile afin de déterminer leur statut légal pose de nombreuses questions, l'éthique peut être remise en cause et la méthodologie utilisée est faussée.

\section{Références}

1 Greulich WW, Pyle SI. Radiographic Atlas of Skeletal Development of the Hand and Wrist, 2nd edition. Stanford, CA: Stanford University Press, 1959.

2 Nowotny T, Eisenberg W, Mohnike K. Unbegleitete minderjährige Flüchtlinge - Strittiges Alter - strittige Altersdiagnostik. Deutsches Ärzteblatt 2014; 111(18); A-786-8.

3 Aynsley-Green A, Cole TJ, Crawley H, Lessof N, Boagj LR, Wallace LMM. Medical, statistical, ethical and human rights considerations in the assessment of age in children and young people sub ject to immigration control. British Medical Bulletin 2012; 1-26.

4 Smith T, Brownlees L. Age assessment practices: a literature review \& annotated bibliography 2011 by United Nations Children's Fund (UNICEF), New York, 2011.

5 Ontell FK, Ivanovic M, Ablin DS, Barlow TW. Bone Age in Children of Diverse Ethnicity. AJR 1996;167; 1395-8. 